\title{
Evaluation of CD40, its ligand CD40L and Bcl-2 in psoriatic patients
}

\author{
Hanna Myśliwiec ${ }^{1}$, Iwona Flisiak ${ }^{1}$, Anna Baran ${ }^{1}$, Maria Górska² ${ }^{2}$ Bożena Chodynicka ${ }^{1}$ \\ ${ }^{1}$ Department of Dermatology and Venereology, Medical University of Bialystok, Poland \\ ${ }^{2}$ Department of Endocrinology, Diabetology and Internal Diseases, \\ Medical University of Bialystok, Poland
}

\begin{abstract}
Psoriasis is a chronic, recurrent, inflammatory disease. Recent investigations indicate an autoimmune pathogenesis of the disease. Apoptosis plays an important role in the regulation of immune mechanisms in many autoimmune diseases. Although CD40, CD40L, and Bcl-2 have already been studied in psoriatic skin lesions, little is known about their circulating forms. The aim of the present study was to evaluate the serum concentrations of $\mathrm{Bcl}-2$, soluble $\mathrm{CD} 40$ and $\mathrm{CD} 40 \mathrm{~L}$ in psoriatic patients. The study was performed using ELISA kits in 39 psoriatic patients before treatment and after two weeks of topical ointment. Data was analyzed with respect to severity of psoriasis, duration of the disease, and coexisting psoriatic arthritis. Our results revealed that serum concentrations of soluble CD40 and CD40L before and after treatment were significantly higher $(\mathrm{p}<0.01$ and $\mathrm{p}<0.001$ ) in patients with psoriasis compared to the control group. Topical treatment of psoriatic lesions with dithranol ointment failed to decrease serum of CD40 and CD40L, which has not been described until now. There was no significant difference in serum Bcl-2 concentration between the compared groups. We did not find significant differences in serum concentrations of Bcl-2, CD40 or CD40L between patients with mild or severe psoriasis, nor any correlation between disease duration and the presence of psoriatic arthritis symptoms. Our data indicates upregulation of the CD40/CD40L system in psoriatic patients despite topical treatment and suggests their possible role in the pathogenesis of psoriasis. (Folia Histochemica et Cytobiologica 2012, Vol. 50, No. 1, 75-79)
\end{abstract}

Key words: psoriasis, Bcl-2, CD40, CD40L

\section{Introduction}

Psoriasis, which affects $2-3 \%$ of the population, is a chronic, recurrent inflammatory disease. Its main feature is excessive proliferation of keratinocytes and disturbances in their differentiation [1]. Recent studies suggest an autoimmune pathogenesis of psoriasis, with involvement of inflammatory mediators derived from lymphocytes $\mathrm{T}$ localized in the skin [2]. The activation of $\mathrm{T}$ lymphocytes depends

Correspondence address: $\mathrm{H}$. Myśliwiec,

Department of Dermatology and Venereology,

Medical University of Bialystok;

Zurawia Str. 14, 15-540 Bialystok, Poland;

tel.: + 48857409566 ;

e-mail: haniam@umb.edu.pl on cytokines released from antigen presenting cells or due to autoimmune processes. Also, in an experimental study on psoriasis in mice, lymphocytes $T$ were shown to play a crucial role in initiation of the disease $[2,3]$.

The protein CD40 is a member of the TNF- $\alpha$ receptor superfamily. CD40 is found on the antigen presenting cells and endotheliocytes. The ligand to CD40, i.e. CD40L (CD154) is present mainly on lymphocytes $\mathrm{T} \mathrm{CD}^{+}$and thrombocytes. The interaction between CD40 and its ligand drives the inflammatory reaction, enhances proinflammatory cytokines (TNF- $\alpha$, IL-12) secretion, promotes T cells survival and their differentiation to type Th1 [4]. CD40 is also an important co-stimulating molecule necessary for antibodies synthesis in lymphocytes B [5]. Lately, CD40/CD40L system activation has been demonstrat- 
ed in many chronic immune diseases, such as subacute skin lupus erythematosus [6], systemic lupus erythematosus [7], dermatomyositis [8], Sjögren's syndrome [9] and ulcerative colitis [10]. So far, the role of $\mathrm{CD} 40 / \mathrm{CD} 40 \mathrm{~L}$ in psoriasis has not been clarified. In one study, an increase in serum CD40 and CD40L in patients with psoriatic arthritis has been demonstrated [11].

One of the main mechanisms responsible for the elimination of excess cells is apoptosis, the programmed cell death. Decreased apoptosis has been described as an important pathomechanism of many immune system diseases. Uncovering the influence of different methods of psoriasis treatment on the apoptosis of keratinocytes and $\mathrm{T}$ lymphocytes is the aim of an increasing number of studies [12]. Methotrexate and UVB radiation $(312 \mathrm{~nm})$ have been demonstrated to induce $\mathrm{T}$ lymphocyte apoptosis within psoriatic lesions [13, 14].

$\mathrm{Bcl}-2$ is a protooncogene protecting the cells against apoptosis. Patients with systemic lupus erythematosus [15] and Behçet disease [16] have been reported to have excessive expression of Bcl-2 in serum and lymphatic cells. To date, there have been no studies on the importance of Bcl-2 concentration in the serum in patients with psoriasis.

We hypothesized that system CD40/CD40L is enhanced in psoriasis, which might reflect activation of T lymphocytes, and at the same time, we expected inhibition of apoptosis. The aim of the present study was to assess the concentrations of soluble CD40, its ligand $\mathrm{CD} 40 \mathrm{~L}$ and $\mathrm{Bcl}-2$ in the serum of patients with psoriasis of varying intensity, before treatment and after two weeks of topical ointment use.

\section{Material and methods}

39 patients (14 women and 25 men) aged 18 to 84 , (mean $48.6 \pm 16.0$ years) with exacerbated plaque-type psoriasis were included in the study. Patients with other health conditions that could affect Bcl-2, CD40, or CD40L serum concentration were excluded from the study. The duration of psoriasis varied from two weeks to 52 months (mean: $18.4 \pm$ \pm 11.8 months). The patients were treated topically: initially with $5 \%$ salicyl ointment, followed by preparations of cignolin (dithranol) in increasing concentrations starting from $0.05 \%$ to $0.3 \%$. Dithranol ointment was prepared in the hospital pharmacy on $3 \%$ salicylic acid (as preservative) and white vaseline as a base. The ointment was applied once a day, for two hours. The intensity of psoriasis before and after two weeks of treatment was assessed using the PASI (Psoriasis Area and Severity Index) score according to Fredriksson and Pettersson [17], which is still widely use to estimate severity of the disease $[18,19]$. The erythema, infiltration and desquamation were separately estimated on the head, trunk, upper and lower limbs. The intensity of each symptom was scored from 0 (no involvement) to 4 (very severe), and the affected area of each region from 0 (no involvement) to $6(90-100 \%)$. The score was obtained by multiplying the sum of intensity scores by each area score, multiplied by constant value (head 0.1 , upper limbs 0.2 , trunk 0.3 , lower limbs 0.4). The total PASI score is the sum of scores of particular body parts. The PASI score varies from 0 to 72 . PASI score before treatment differed from 2.8 to 27.6 (mean $10.9 \pm 6.3$ ). The control group consisted of ten healthy subjects: eight females and two males, mean age $41 \pm$ \pm 16.0 years.

Blood was taken (in the morning) twice: before and after two weeks of topical treatment. After centrifugation, the serum was stored at $-80^{\circ} \mathrm{C}$ until analyses. The concentrations of soluble CD40, CD40L and Bcl-2 in serum were measured using commercially available ELISA kits: CD40 and CD40L (Bender MedSystems, Vienna, Austria, sensitivity respectively $12 \mathrm{pg} / \mathrm{ml}$ and $0.062 \mathrm{ng} / \mathrm{ml}$; intra-assay coefficience of variation [CV] $5.5 \%$ and $6.8 \%$ ) and $\mathrm{Bcl}-2$ (Bender MedSystems, Vienna, Austria, sensitivity $0.5 \mathrm{ng} / \mathrm{ml}$; CV 8.6\%).

Statistical analysis was performed using Mann-Whitney and Wilcoxon tests, $\mathrm{p}<0.05$ was regarded as significant. The correlations between the variables were calculated using Spearman's test.

The study was approved by the Bioethical Committee of the Medical University of Bialystok.

\section{Results}

The concentrations of soluble CD40 and CD40L in the studied group before treatment were significantly higher than in the control group (Table 1). After two weeks of treatment, despite a halving in the PASI score (from $10.9 \pm 6.3$ to $4.2 \pm 3.4$ ), the concentrations of soluble CD40 and CD40L remained significantly higher than in the control group. Neither CD40 nor CD40L concentrations changed significantly after treatment. The concentration of Bcl-2 in the control group was higher than in psoriatic patients before treatment and after treatment (Table 1), but no significant differences between the groups were found. We found no significant correlations between CD40, $\mathrm{CD} 40 \mathrm{~L}$ or Bcl-2 and severity of psoriasis, its duration or psoriatic arthritis symptoms.

\section{Discussion}

In the present study, we demonstrate for the first time the increase in both soluble CD40 and CD40L in patients suffering from psoriasis even without arthritis. The concentrations were significantly higher compared to the control group both before treatment and after two weeks of topical ointment use. The higher 
Table 1. Concentrations of CD40, CD40L and Bcl-2 in serum of the control group and patients suffering from psoriasis before and after treatment. Data is given as median (full range). Significant differences compared to the control group are shown as $* * p<0.01 ; * * \mathrm{p}<0.001$ within a row

\begin{tabular}{|l|c|c|c|}
\hline \multirow{2}{*}{} & \multirow{2}{*}{ Control group } & \multicolumn{2}{|c|}{ Psoriasis } \\
\cline { 3 - 4 } & & Before treatment & After treatment \\
\hline SCD40 $[\mathrm{pg} / \mathbf{m l}]$ & $33(12-48)$ & $55(18-108)^{* *}$ & $57(19-101)^{* *}$ \\
\hline SCD40L $[\mathbf{n g} / \mathbf{m l}]$ & $2.2(0.6-7.2)$ & $10.4(2.9-19.4)^{* * *}$ & $10.2(4.9-19.7)^{* * *}$ \\
\hline Bcl-2 $[\mathbf{n g} / \mathbf{m l}]$ & $15.0(7.0-76.5)$ & $11.1(5.6-20.2)$ & $11.1(6.7-23.8)$ \\
\hline
\end{tabular}

concentrations of soluble CD40 and CD40L were noted independently of the severity of skin lesions (PASI score) and the duration of the disease.

CD40/CD40L system is an important factor in the pathogenesis of autoimmune disorders, being a key mediator in T and B lymphocytes cross talk [20]. CD40 is a membrane receptor appearing on B cells after stimulation by cytokines derived from T lymphocytes [21]. Binding of CD40L to CD40 enables the transmission of a co-stimulating signal, leading to enhanced proliferation, differentiation and activation of B lymphocytes, as well as synthesis of antibodies [22]. CD40/CD40L system has been reported to be an important factor in maintaining the autoimmune process [23]. Enhanced expression of CD40L on T lymphocytes and the increase in its concentration in serum was associated with an increase in specific antibodies and the clinical activity of active lupus erythematosus and rheumatoid arthritis [24, 25]. The presence of CD40/CD40L was shown on keratinocytes both in normal skin and in psoriatic skin lesions [26]. The enhanced expression of CD40L was associated with early stage of the disease [26].

We report significantly higher concentration of soluble CD40 and CD40L in psoriatic patients compared to the control group, which could indicate their role in the pathogenesis of psoriasis. The results of the present study are in accordance with those of previous reports into psoriatic arthritis. Daoussis et al. [11] obtained similar data, demonstrating also increased expression of CD40L on activated $\mathrm{T}$ lymphocytes derived from patients with psoriatic arthritis. Additionally, the authors observed in vitro the decrease in CD40L on T lymphocytes treated with cyclosporin A [11], which seems to confirm this theory. Since several studies in recent years have shown expression of the studied molecules on thrombocytes and endotheliocytes and their important role in the development of atherosclerosis and thrombosis [27, 28], it might at least partly explain high prevalence of cardiovascular diseases in patients suffering from psoriasis. The fact that topical treatment does not lead to a decrease in CD40/CD40L pathway activity is worrying. This might mean that despite regression of skin lesions, the ongoing inflammatory process predisposes the patients with psoria- sis (independently of intensity and duration of disease) to cardiovascular comorbidities.

This is the first report indicating that patients treated with topical ointments and untreated psoriatic patients have similar CD40/CD40L system upregulation. We propose that they need systemic treatment. Since the number of analyzed patients in our study was small, evaluation of a larger cohort of psoriatic patients in future studies is desirable.

On the other hand, our finding of upregulation of CD40/CD40L in psoriasis, together with previous reports on efficacy of anti-CD40L antibodies in the treatment of autoimmune disorders, suggests the need to conduct such studies also in psoriasis. Toubi et al. demonstrated the therapeutic potential of inhibiting CD40/CD40L in lupus glomerulonephritis. According to them, such inhibition prevents synthesis of antibodies and deposition of immune complexes in renal glomeruli of patients suffering from systemic lupus erythematosus [29].

Contrary to our expectations, serum concentration of Bcl-2 in psoriatic patients was not higher than in the control group. Bcl-2 protein is a product of the bcl-2 gene localized on the short arm of chromosome 18 and exerts an antiapoptotic role [30]. Increased serum expression of Bcl-2 has been reported in patients with systemic lupus erythematosus [15] and in Behçet disease [16]. So far, there has been little research on Bcl-2 expression in psoriatic epidermis. Most authors have concentrated on apoptosis of keratynocytes, but not lymphocytes [7, 31]. Avramidis et al. [32] found significant reduction in Bcl-2 and induction of apoptosis in endothelial cells of patients with psoriasis during etanercept treatment. Yildiz et al. [33] observed enhanced expression of bcl-2 gene in lymphocytes within the epidermis of psoriatic patients. The authors suggested that prolonged survival of epidermal lymphocytes, due to their resistance against apoptosis, could be the reason for the chronic course of the disease, its recurrences and resistance to treatment. The decreased $\mathrm{Bcl}-2$ expression in lymphocytes within psoriatic lesions after topical treatment with calcipotriol seems to confirm this theory, although calcipotriol appears to have its effect main- 
ly on keratinocytes rather than lymphocytes [34]. Our results do not show any significant difference in serum concentration of $\mathrm{Bcl}-2$ in patients with psoriasis, either before or after topical treatment, compared to the control group. A low level of Bcl-2 is an unexpected finding and may suggest that $\mathrm{Bcl}-2$ does not play a major contributory role in the antiapoptotic mechanisms that are proposed to be important in psoriasis. Nevertheless, there remains the possibility that other antiapoptotic proteins might participate in the hypothetical blockage of the apoptotic response in psoriatic lymphocytes. Further studies are necessary to clarify this issue.

In conclusion, in this study we have not observed any significant difference either in Bcl-2 concentration in the serum of psoriatic patients, nor an effect of the topical treatment on its expression.

The results of the present study indicate activation of CD40/CD40L signaling pathway in patients suffering from psoriasis independently of the severity of skin lesions, duration of the disease or topical treatment. This seems to confirm the role of CD40/ /CD40L interaction in the pathogenesis of psoriasis. Unfortunately, we also certified a lack of topical treatment effect on the immune mechanisms involved in the disease, which indicates the need for systemic treatment. Further investigations are needed to confirm their role in an increased risk of cardiovascular comorbidities among those patients.

\section{Acknowledgements}

This study was supported by a grant from the Medical University of Bialystok, Poland.

\section{References}

1. Nickloff BJ, Griffiths CE. Lymphocyte trafficking in psoriasis: a new perspective emphasizing the dermal dendrocyte with active dermal recruitment mediated via endothelial cells followed by intra-epidermal T-cell activation. J Invest Dermatol. 1990;95:35-37.

2. Gilhar A, David M, Ullmann Y, Berkutski T, Kalish RS. T-lymphocyte dependence of psoriatic pathology in human psoriatic skin grafted to SCID mice. J Invest Dermatol. 1997;109:283-288.

3. Wrone-Smith T, Nickoloff BJ. Dermal injection of immunocytes induces psoriasis. J Clin Invest. 1996;98:1878-1887.

4. Howland KC, Ausubel LJ, London CA, Abbas AK. The roles of CD28 and CD40 ligand in T cell activation and tolerance. J Immunol. 2000;164:4465-4470.

5. Bennett SR, Carbone FR, Karamalis F, Flavell RA, Miller JF, Heath WR. Help for cytotoxic-T-cell responses is mediated by CD40 signalling. Nature. 1998;393:478-480.

6. Caproni M, Torchia D, Antiga E et al. The CD40/CD40 Ligand system in the skin of patients with subacute cutaneous lupus erythematosus. J Rheumatol. 2007;34:2412-2416 .
7. Toubi E, Schoenfeld Y. The role of CD40-CD154 interactions in autoimmunity and the benefit of disrupting this pathway. Autoimmunity. 2004;37:457-464.

8. Caproni M, Torchia D, Cardinali $\mathrm{C}$ et al. Infiltrating cells, related cytokines and chemokine receptors in lesional skin of patients with dermatomyositis. Br J Dermatol. 2004;151: 784-791.

9. Nakamura H, Kawakami A, Tominaga M, Migita K, Kawabe Y, Nakamura T. Expression of CD40/CD40 ligand and Bcl-2 family proteins in labial salivary glands of patients with Sjögren's syndrome. Lab Invest. 1999;79:261-269.

10. Danese S, Katz JA, Saibeni S et al. Activated platelets are the source of elevated levels of soluble CD40 ligand in the circulation of inflammatory bowel disease patients. Gut. 2003;52:1435-1441.

11. Daoussis D, Antonopoulos I, Andonopoulos AP, Liossis SNC. Increased expression of CD154 (CD40L) on stimulated T-cells from patients with psoriatic arthritis. Rheumatology. 2007;46:227-231.

12. Murphy FP, Coven TR, Burack LH et al. Clinical clearing of psoriasis correlates with cutaneous T-cell depletion via apoptosis: evidence for selective effects on activated $\mathrm{T}$ lymphocytes. Arch Dermatol. 1999;135:1495-1502.

13. Ozawa M, Ferenczi K, Kikuchi T et al. 312-nanometer ultraviolet B light (narrow-band UVB) induces apoptosis of T cells within psoriatic lesions. J Exp Med. 1999;189:711-718.

14. Heenen M, Laporte M, Noel JC, de Graef C. Methotrexate induces apoptotic cell death in human keratinocytes. Arch Dermatol Res. 1998;290:240-245.

15. Miret C, Font J, Molina R et al. Bcl-2 oncogen levels correlate with systemic lupus erythematosus disease activity. Anticanser Res. 1999;19:3071-3076.

16. Senturk N, Yildiz L, Sullu Y, Kandemir B, Turanli AY. Expression of bcl-2 protein in active skin lesions of Behcet's disease. Int J Dermatol. 2001;40:747-750.

17. Fredriksson T, Petterson U. Severe psoriasis - oral therapy with a new retinoid. Dermatologica. 1978;157:238-244.

18. Schmitt J, Wozel G. The Psoriasis area and severity index is the adequate criterion to define severity in chronic plaquetype psoriasis. Dermatology. 2005;210:194-199.

19. Langley RG, Ellis CN. Evaluating psoriasis with psoriasis area and severity index, psoriasis global assessment, and lattice system physician's global assessment. J Am Acad Dermatol. 2004;51:563-569.

20. Pérez-Melgosa M, Hollenbaugh $\mathrm{D}$, Wilson CB. CD40 ligand is a limiting factor in the humoral response to T cell-dependent antigens. J Immunol. 1999;163:1123-1127.

21. Duhen T, Pasero C, Mallet F, Barbarat B, Olive D, Costello RT. LIGHT costimulates CD40 triggering and induces immunoglobulin secretion; a novel key partner in T cell-dependent B cell terminal differentiation. Eur J Immunol. 2004;34:3534-3541 .

22. Elzey BD, Grant JF, Sinn HW, Nieswandt B, Waldschmidt TJ, Ratliff TL. Cooperation between platelet-derived CD154 and $\mathrm{CD}^{+}{ }^{+} \mathrm{T}$ cells for enhanced germinal center formation. $J \mathrm{Leu}$ koc Biol. 2005;78:80-84.

23. Dadgostar H, Zarnegar B, Hoffmann A et al. Cooperation of multiple signaling pathways in CD40-regulated gene expression in B lymphocytes. Proc Natl Acad Sci USA. 2002;99:1497-1502.

24. Komura K, Sato S, Hasegawa M, Fujimoto M, Takehara K. Elevated circulating CD40L concentrations in patients with systemic sclerosis. J Rheumatol. 2004;31:514-519.

25. Saijo S, Asano M, Horai R, Yamamoto H, Iwakura Y. Suppression of autoimmune arthritis in interleukin-1-deficient 
mice in which $\mathrm{T}$ cell activation is impaired due to low levels of CD40 ligand and OX40 expression on T cells. Arthritis Rheum. 2002;46:533-544.

26. Ohta Y, Hamada Y. In situ expression of CD40 and CD40 ligand in psoriasis. Dermatology. 2004;209:21-28.

27. Tousoulis D, Antaniades C, Koumallos N, Stefanadis C. Proinflammatory cytokines in acute coronary syndromes: from bench to bedside. Cytokine Growth Factor Rev. 2006;17: 225-233.

28. Anand SX, Viles-Gonzalez JF, Badimon JJ, Cavusoglu E, Marmur MJ. Membrane-associated CD40L and SCD40L in atherothrombotic disease. Thromb Haemost. 2003;90: 377-384.

29. Toubi E, Shoenfeld Y. The role of CD40-CD154 interactions in autoimmunity and the benefit of disrupting this pathway. Autoimmunity. 2004;37:457-464.
30. Hockenbery D, Nunez G, Milliman C, Schreiber RD, Korsmeyer SJ. Bcl-2 is an inner mitochondrial membrane protein that blocks programmed cell death. Nature. 1990;22:334-336.

31. Laporte M, Galand P, Fokon D, de Graef C, Heenen M. Apoptosis in established and healing psoriasis. Dermatol. 2000;200:314-316.

32. Avramidis G, Kruger-Krasagakis S, Krasagakis K, Fragiadaki I, Kokolakis G, Tosca A. The role of endothelial cell apoptosis in the effect of etanercept in psoriasis. Br J Dermatol. 2010;163:928-934.

33. Yildiz L, Baris S, Senturk N, Kandemir B. Overexpression of bcl-2 in lymphocytes of psoriatic skin. J Eur Acad Dermatol Venereol. 2003;17:538-540.

34. El-Domvati M, Barakat M, Abdel-Razek R, El-Din Anbar T. Apoptosis, $\mathrm{P} 53$ and Bcl-2 expression in response to topical calcipotriol therapy for psoriasis. Int J Dermatol. 2007;46:468-474.

Submitted: 21 March, 2011 Accepted after reviews: 14 November, 2011 